\title{
CONTROLLING A NON-HOMOGENEOUS TIMOSHENKO BEAM WITH THE AID OF THE TORQUE
}

\author{
GRIGORY M. SKLYAR，GRZEGORZ SZKIBIEL \\ Institute of Mathematics \\ Szczecin University, ul. Wielkopolska 15, 70-451 Szczecin, Poland \\ e-mail: szkibiel@wmf.univ.szczecin.pl, sklar@univ.szczecin.pl
}

\begin{abstract}
Considered is the control and stabilizability of a slowly rotating non-homogeneous Timoshenko beam with the aid of a torque. It turns out that the beam is (approximately) controllable with the aid of the torque if and only if it is (approximately) controllable. However, the controllability problem appears to be a side-effect while studying the stabilizability. To build a stabilizing control one needs to go through the methods of correcting the operators with functionals so that they have finally the appropriate form and the results on $C^{0}$-continuous semigroups may be applied.
\end{abstract}

Keywords: Timoshenko beam, rotating beam control, approximate control, stabilizability.

\section{Introduction}

We consider the following system of equations describing the rotating Timoshenko beam (see Sklyar and Szkibiel, 2008a; 2008b):

$$
\begin{aligned}
\varrho(x) \ddot{w}(x, t)- & \left(K(x)\left(w^{\prime}(x, t)+\xi(x, t)\right)^{\prime}\right. \\
= & -\varrho(x)(x+r) \ddot{\theta}(t), \\
R(x) \ddot{\xi}(x, t)- & \left(E(x) \xi^{\prime}(x, t)\right)^{\prime} \\
& +K(x)\left(w^{\prime}(x, t)+\xi(x, t)\right) \\
= & R(x) \ddot{\theta}(t) .
\end{aligned}
$$

The beam is clamped to a rotating disk propelled be an engine. By $r$ we denote the radius of the disc while $\theta=$ $\theta(t)$ is the rotation angle considered a function of time $t \geq 0$. To a (uniform) cross section of the beam at point $x$, with $0 \leq x \leq 1$, we assign the following: $E(x)$, which is the flexural rigidity, $K(x)$ - shear stiffness, $\varrho(x)$-mass of the cross section and $R(x)$-rotary inertia. All of the above functions are assumed to be real and bounded by two positive numbers. We also assume that their first and second derivatives are bounded. The beam is normalized and its length is assumed to be 1 .

We denote by $w(x, t)$ the deflection of the center line of the beam (Fig. 1) and by $\xi(x, t)$ the rotation angle of the cross section area at the location $x$ and at the time $t$ (Fig. 2). Finally, $g^{\prime}(x, t)$ denotes the partial derivative with respect to $x$ and $\dot{g}(x, t)$ the partial derivative with respect to $t$ for any function $g$ of two variables. We use

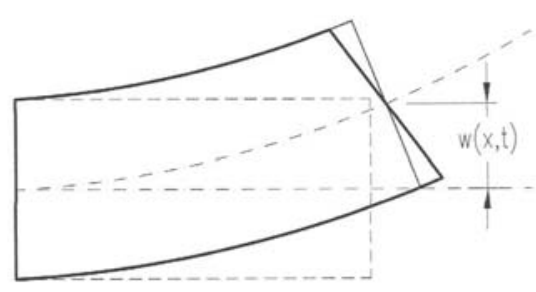

Fig. 1. Deflection of the center line.

this notation also for functions of one variable depending on whether they are considered functions of $x$ or $t$.

Assume that there is no deformation at the clamped end. As a consequence of the energy balance law, we obtain the following boundary conditions for $t \geq 0$ :

$$
\begin{aligned}
w(0, t)=\xi(0, t) & =0, \\
w^{\prime}(1, t)+\xi(1, t)=\xi^{\prime}(1, t) & =0 .
\end{aligned}
$$

The control of the system is realized by a function $u$ being simply the angular acceleration $(u(t)=\ddot{\theta}(t))$ of the disk. It is shown (Krabs and Sklyar, 2002) that the homogeneous beam is approximately controllable. Also, it is proved that the exact controllability from the position of rest to an arbitrary position is possible provided that the parameter functions $(\varrho, R, K$ and $E$ ) satisfy some 


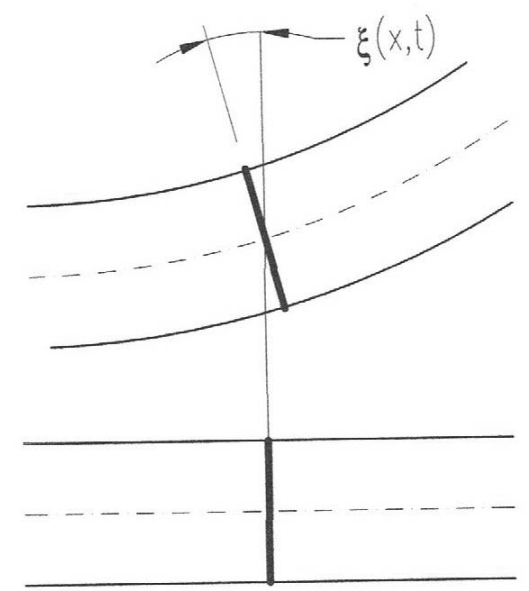

Fig. 2. Rotation angle the cross section area.

conditions like those given by Sklyar and Szkibiel (2008a; 2007).

Krabs and Sklyar (2002) also consider the concept of controllability with the aid of the torque, and they show that the (homogeneous) system is approximately controllable with the aid of the torque if it is approximately controllable in the usual sense. We shall show that this is the case also for a non-homogeneous beam.

Let $I_{d}$ denote the disk inertia. The control (with the aid of the torque) $\bar{u}$ is given by the equation

$$
\begin{aligned}
\bar{u}(t)= & I_{d} \ddot{\theta}(t) \\
& +\int_{0}^{1} \varrho(x)(x+r)(\ddot{w}(x, t)+(x+r) \ddot{\theta}(t)) \mathrm{d} x \\
& -\int_{0}^{1} R(x)(\ddot{\xi}(x, t)-\ddot{\theta}(t)) \mathrm{d} x .
\end{aligned}
$$

After elementary transformations of (1) and integration by parts with the use of the boundary conditions (2), Eqn. (3) simplifies to

$$
\bar{u}(t)=I_{d} \ddot{\theta}(t)+E(0) \xi^{\prime}(0, t)-r K(0) w^{\prime}(0, t) .
$$

To state the problem of controllability, we describe the initial and final positions (or states) of the beam by

$$
\left(w_{0}, \dot{w}_{0}, \xi_{0}, \dot{\xi}_{0}, \theta_{0}, \dot{\theta}_{0}\right)^{T}
$$

and

$$
\left(w_{T}, \dot{w}_{T}, \xi_{T}, \dot{\xi}_{T}, \theta_{T}, \dot{\theta}_{T}\right)^{T}
$$

respectively, where $w_{0}, \dot{w}_{0}, \xi_{0}, \dot{\xi}_{0}, w_{T}, \dot{w}_{T}, \xi_{T}, \dot{\xi}_{T}$ are some (complex) functions of variable $x \in[0,1]$ and $\theta_{0}$, $\dot{\theta}_{0}, \theta_{T}, \dot{\theta}_{T}$ are some complex numbers. For simplicity, we assume that, at the beginning, the beam is at the position of rest, i.e., $w_{0}=\dot{w}_{0}=\xi_{0}=\dot{\xi}_{0}=0$ and $\theta_{0}=\dot{\theta}_{0}=0$.

The problem of (exact) controllability is to find a control function $u:[0, T] \rightarrow \mathbb{C}$ such that the solution $(w, \xi)^{T}$ of (1)-(2) satisfies

$$
\begin{aligned}
w(x, 0) & =0, & \dot{w}(x, 0) & =0, \\
\xi(x, 0) & =0, & \dot{\xi}(x, 0) & =0, \\
w(x, T) & =w_{T}(x), & \dot{w}(x, T) & =\dot{w}_{T}(x), \\
\xi(x, T) & =\xi_{T}(x), & \dot{\xi}(x, T) & =\dot{\xi}_{T}(x), \\
\theta(0) & =0, & \dot{\theta}(0) & =0, \\
\theta(T) & =\theta_{T}, & \dot{\theta}(T) & =\dot{\theta}_{T},
\end{aligned}
$$

and $u(t)=\ddot{\theta}(t)$ for $t \in[0, T]$.

The problem of (exact) controllability with the aid of the torque is to find a control $\bar{u}:[0, T] \rightarrow \mathbb{C}$ such that the solution $(w, \xi)^{T}$ of (1)-(2) satisfies (5) and Eqn. (4) is satisfied for each $t \in[0, T]$.

In practice, finding the control is a rather difficult task and most of the authors decide rather to express the control in terms of its Fourier series coefficients (Russell, 1967; Sklyar and Szkibiel, 2008a) or just prove merely its existence (Avdonin and Ivanov, 1995; Avdonin and Moran, 2001). The approach presented by the latter authors is based on the paper by Levin (1961) and allows stating an equivalent condition for the existence of control in terms of analytic functions. Yet another method of expressing the control function is the approximation via numerical analysis. It is considered for the more general class of hyperbolic equations by, e.g., Respondek (2008) and Zerrik et al. (2007).

While dealing with our approach the question that arises is what possible final states may be achieved using any control (Sklyar and Szkibiel, 2012). If the set of those states is dense in the target space, we call the beam $a p$ proximately controllable. If we are able to find (or prove the existence) of the functional

$$
p(w, \xi, \theta, \dot{w}, \dot{\xi}, \dot{\theta})
$$

such that every solution $(w, \xi, \theta)^{T}$ of (1)-(3) with feedback control

$$
\bar{u}(t)=p(w(\cdot, t), \xi(\cdot, t), \theta(t), \dot{w}(\cdot, t), \dot{\xi}(\cdot, t), \dot{\theta}(t))
$$

causes the extinguishing of the total energy of the system, i.e.,

$$
\begin{array}{r}
\int_{0}^{1} w^{\prime}(x, t)^{2} \mathrm{~d} x, \int_{0}^{1} \dot{w}(x, t)^{2} \mathrm{~d} x \rightarrow 0 \\
\int_{0}^{1} \xi^{\prime}(x, t)^{2} \mathrm{~d} x, \int_{0}^{1} \dot{\xi}(x, t)^{2} \mathrm{~d} x \rightarrow 0 \\
\theta(t), \dot{\theta}(t) \rightarrow 0
\end{array}
$$

as $t \rightarrow \infty$, then we call the system strongly stabilizable. We notice that the the concept of strong stabilizability is 
utilized also in discrete systems: see, e.g., the work of Kaczorek (2012) and the reference therein. Also, one can clearly see that strongly stabilizable objects are depicted by systems of equations that are stable or exponentially stable in the sense of $C_{0}$-continuous semigroup theory, see the work of Ostalczyk (2012) for the latest achievements in the study of this theory applications.

\section{Related spaces and operators}

We consider the set $H$ of pairs of functions from $L^{2}(0,1)$ whose second derivatives are again in $L^{2}(0,1)$. In addition, we shall deal with the inner product defined by the formula

$$
\begin{aligned}
\left\langle\left(\begin{array}{l}
f_{1} \\
f_{2}
\end{array}\right),\left(\begin{array}{l}
g_{1} \\
g_{2}
\end{array}\right)\right\rangle= & \frac{1}{I_{d}} \int_{0}^{1} \varrho(x) f_{1}(x) \overline{g_{1}(x)} \mathrm{d} x \\
& +\frac{1}{I_{d}} \int_{0}^{1} R(x) f_{2}(x) \overline{g_{2}(x)} \mathrm{d} x .
\end{aligned}
$$

The functions $\varrho$ and $R$ are real functions defined in the previous section and the disc inertia $I_{d}$ is a positive real number. Therefore the inner product (7) is equivalent to the standard one. On the set

$$
D(A)=\left\{\left(\begin{array}{l}
y \\
z
\end{array}\right) \in H: \begin{array}{l}
y(0)=z(0)=0 \\
y^{\prime}(1)+z(1)=z^{\prime}(1)=0
\end{array}\right\}
$$

we define the operator $A$ with values in the set $L^{2}(0,1)^{2}$ by the formula

$$
A\left(\begin{array}{l}
y \\
z
\end{array}\right)=\left(\begin{array}{c}
-(1 / \varrho)\left(K\left(y^{\prime}+z\right)\right)^{\prime} \\
-(1 / R)\left(E z^{\prime}\right)^{\prime}+(1 / R) K\left(y^{\prime}+z\right)
\end{array}\right) .
$$

One can notice that $D(A)$ is dense in $H$ and $A$ is surjective (Sklyar and Szkibiel, 2008b). Also, it is proved there that the operator $A$ is self-adjoint, positive and possesses a compact resolvent.

Upon defining $b(x)=\left(\begin{array}{c}-x-r \\ 1\end{array}\right)$, Eqn. (1) takes the operator form

$$
\left(\begin{array}{c}
\ddot{w}(\cdot, t) \\
\ddot{\xi}(\cdot, t)
\end{array}\right)+A\left(\begin{array}{c}
w(\cdot, t) \\
\xi(\cdot, t)
\end{array}\right)=b(\cdot) \ddot{\theta}(t) .
$$

Thus we arrive at an abstract wave equation with a distribution control $u=\ddot{\theta}$ (Krabs and Sklyar, 2002).

We shall state a similar equation for the distributive control with the aid of the torque. With the use of the inner product definition (7), integration by parts and boundary conditions (2), we arrive at

$$
I_{d}\left\langle A\left(\begin{array}{l}
y \\
z
\end{array}\right), b\right\rangle=-r K(0) y^{\prime}(0)+E(0) z^{\prime}(0) .
$$

Therefore (4) may be written in the form

$$
\frac{\bar{u}(t)}{I_{d}}=\ddot{\theta}(t)+\left\langle A\left(\begin{array}{c}
w(\cdot, t) \\
\xi(\cdot, t)
\end{array}\right), b\right\rangle .
$$

This and (8) lead to the following equation:

$$
\left(\begin{array}{c}
\ddot{w}(\cdot, t) \\
\ddot{\xi}(\cdot, t)
\end{array}\right)+A\left(\begin{array}{c}
w(\cdot, t) \\
\xi(\cdot, t)
\end{array}\right)+\left\langle A\left(\begin{array}{c}
w(\cdot, t) \\
\xi(\cdot, t)
\end{array}\right), b\right\rangle b=\frac{b}{I_{d}} \bar{u}(t) .
$$

In the above, the self-adjoint operator $A$ is disturbed by a functional. Hence we use some technics like the one given by Krabs and Sklyar (2002) to arrive at an equation like (8). We start with defining the operator $\bar{A}$ by the block matrix

$$
\bar{A}=\left(\begin{array}{ll}
A & 0 \\
0 & 0
\end{array}\right) \text {. }
$$

Then $\bar{A}: D(\bar{A}) \rightarrow \bar{H}$, where $D(\bar{A})=D(A) \times \mathbb{C}$ and $\bar{H}=H \times \mathbb{C}$. Additionally, we set $\bar{b} \in \bar{H}$ to be

$$
\left(\begin{array}{l}
b \\
1
\end{array}\right)=\left(\begin{array}{c}
-x-r \\
1 \\
1
\end{array}\right)
$$

and define $\tilde{u}(t)$ to be equal to $\bar{u}(t) / I_{d}$. formula

Next we define the inner product on $\bar{H}$ by the

$$
\left\langle\left(\begin{array}{l}
X_{1} \\
\alpha_{1}
\end{array}\right),\left(\begin{array}{c}
X_{2} \\
\alpha_{2}
\end{array}\right)\right\rangle_{\bar{H}}=\left\langle X_{1}, X_{2}\right\rangle+\alpha_{1} \overline{\alpha_{2}},
$$

where $\langle\cdot, \cdot\rangle$ is the "old" inner product (i.e., the one defined by (7)) and $\bar{\alpha}$ is the complex conjugate.

Let $I$ be the identity operator and $\bar{b}^{*}$ the conjugate of $\bar{b}$, i.e., $\bar{b}^{*} Z=\langle Z, \bar{b}\rangle_{\bar{H}}$ for any $Z \in \bar{H}$. Then (9) and (10) may be put together and written as

$$
\ddot{Y}(t)+\left(I+\bar{b} \bar{b}^{*}\right) \bar{A} Y(t)=\bar{b} \tilde{u}(t),
$$

where

$$
Y(t)=\left(\begin{array}{c}
w(\cdot, t) \\
\xi(\cdot, t) \\
\theta(t)
\end{array}\right)
$$

We redefine the inner product (11) in the following way:

$$
\langle Y, Z\rangle_{1}=\left\langle\left(I+\bar{b} \bar{b}^{*}\right)^{-1 / 2} Y,\left(I+\bar{b} \bar{b}^{*}\right)^{-1 / 2} Z\right\rangle_{\bar{H}} .
$$

We notice that the above inner product is equivalent to the one defined in (11). Finally, we define the operator $\widetilde{A}: D(\bar{A}) \rightarrow \bar{H}$ by the formula $\widetilde{A}=\left(I+\bar{b} \bar{b}^{*}\right) \bar{A}$, so Eqn. (12) takes the form of an abstract wave equation with a distribution control:

$$
\ddot{Y}(t)+\widetilde{A} Y(t)=\bar{b} \tilde{u}(t) .
$$

Further on we discover basic properties of $\widetilde{A}$. Actually, the way the inner product $\langle\cdot, \cdot\rangle_{1}$ and the operator $\widetilde{A}$ are defined make all needed properties of $\widetilde{A}$ satisfied. They are contained in the following theorem.

Theorem 1. The operator $\widetilde{A}$ is self-adjoint with respect to $\langle\cdot, \cdot\rangle_{1}$ and has a compact resolvent. 
Proof. We start with showing that $\widetilde{A}$ is positive and symmetric. We shall use the properties of the operator $A$ stated and proved by Sklyar and Szkibiel (2008b). Let $Y_{1}, Z_{1}$ be arbitrary elements of $D(A)$. Hence

$$
Y=\left(\begin{array}{c}
Y_{1} \\
\theta
\end{array}\right), \quad Z=\left(\begin{array}{c}
Z_{1} \\
\tau
\end{array}\right)
$$

are elements of $D(\bar{A})$. Using the definition of the inner products (11), (13) and the self-adjointness of $(I+$ $\left.\bar{b} \bar{b}^{*}\right)^{1 / 2}$, we obtain

$$
\begin{aligned}
\langle\widetilde{A} Y, Y\rangle_{1} & =\left\langle\left(I+\bar{b} \bar{b}^{*}\right) \bar{A} Y, Y\right\rangle_{1} \\
& =\left\langle\left(I+\bar{b} \bar{b}^{*}\right)^{1 / 2} \bar{A} Y,\left(I+\bar{b} \bar{b}^{*}\right)^{-1 / 2} Y\right\rangle_{\bar{H}} \\
& =\langle\bar{A} Y, Y\rangle_{\bar{H}} \\
& =\left\langle A Y_{1}, Y_{1}\right\rangle \geq 0 .
\end{aligned}
$$

Therefore $\widetilde{A}$ is non-negative. Moreover, $\langle\widetilde{A} Y, Y\rangle_{1}=0$ if and only if $Y_{1}=\left(\begin{array}{l}0 \\ 0\end{array}\right)$. Thus $\widetilde{A}$ has one-dimensional kernel, so the eigenspace corresponding to the eigenvector 0 is one-dimensional.

To prove symmetry, we partly repeat the above reasoning and get

$$
\begin{aligned}
\langle\widetilde{A} Y, Z\rangle_{1}=\langle\bar{A} Y, Z\rangle_{\bar{H}} & =\left\langle A Y_{1}, Z_{1}\right\rangle \\
& =\left\langle Y_{1}, A Z_{1}\right\rangle=\langle Y, \widetilde{A} Z\rangle_{1}
\end{aligned}
$$

by the self-adjointness of the operator $A$.

In order to show that $\widetilde{A}$ is self-adjoint, we need to justify the equality $D(\bar{A})=D\left(\widetilde{A}^{*}\right)$, i.e., we need to prove the inclusion $D\left(\widetilde{A}^{*}\right) \subset D(\bar{A})$, as the other one follows by symmetry. For that purpose, we first prove that $D\left(\bar{A}^{*}\right) \subset$ $D(\bar{A})$. To show this, we take an arbitrary $\left(\begin{array}{l}Z_{1} \\ \tau_{1}\end{array}\right) \in D\left(\bar{A}^{*}\right)$ and consider $Y=\left(\begin{array}{c}Y_{1} \\ 0\end{array}\right)$ with $Y_{1} \in D(A)$.

Let

$$
\left(\begin{array}{c}
Z_{2} \\
\tau_{2}
\end{array}\right)=\bar{A} *\left(\begin{array}{c}
Z_{1} \\
\tau_{1}
\end{array}\right) \text {. }
$$

We have

$$
\begin{aligned}
\left\langle Y_{1}, Z_{2}\right\rangle & =\left\langle Y, \bar{A}^{*}\left(\begin{array}{c}
Z_{1} \\
\tau_{1}
\end{array}\right)\right\rangle_{\bar{H}} \\
& =\left\langle\bar{A}\left(\begin{array}{c}
Y_{1} \\
0
\end{array}\right),\left(\begin{array}{c}
Z_{1} \\
\tau_{1}
\end{array}\right)\right\rangle_{\bar{H}}=\left\langle A Y_{1}, Z_{1}\right\rangle
\end{aligned}
$$

Since $A$ is surjective, one may find $Z_{3} \in D(A)$ such that $A Z_{3}=Z_{2}$. Therefore

$$
\bar{A}\left(\begin{array}{c}
Z_{3} \\
0
\end{array}\right)=\left(\begin{array}{c}
Z_{2} \\
0
\end{array}\right) \text {. }
$$

What follows is

$$
\begin{aligned}
\left\langle Y_{1}, Z_{2}\right\rangle & =\left\langle Y,\left(\begin{array}{c}
Z_{2} \\
\tau_{2}
\end{array}\right)\right\rangle_{\bar{H}} \\
& =\left\langle Y, \bar{A}\left(\begin{array}{c}
Z_{3} \\
0
\end{array}\right)\right\rangle_{\bar{H}}=\left\langle A Y_{1}, Z_{3}\right\rangle .
\end{aligned}
$$

Thus

$$
0=\left\langle A Y_{1}, Z_{1}\right\rangle-\left\langle A Y_{1}, Z_{3}\right\rangle=\left\langle A Y_{1}, Z_{1}-Z_{3}\right\rangle .
$$

That implies $Z_{1}=Z_{3}$, so, in particular, $Z_{1} \in D(A)$ and $\left(\begin{array}{c}Z_{1} \\ \tau_{1}\end{array}\right) \in D(\bar{A})$. As for the self-adjointness of $\widetilde{A}$, let us take any $Y \in D(\bar{A})$ and $Z \in D\left(\widetilde{A}^{*}\right)$. As before, we obtain

$$
\left\langle Y, \widetilde{A}^{*} Z\right\rangle_{1}=\langle\widetilde{A} Y, Z\rangle_{1}=\langle\bar{A} Y, Z\rangle_{\bar{H}} .
$$

On the other hand, if $Z^{\prime}=\widetilde{A}^{*} Z$, then

$$
\left\langle Y, \widetilde{A}^{*} Z\right\rangle_{1}=\left\langle Y, Z^{\prime}\right\rangle_{1}=\left\langle Y,\left(I+\bar{b} \bar{b}^{*}\right)^{-1} Z^{\prime}\right\rangle_{\bar{H}} .
$$

Thus, for each $Y \in D(\bar{A})$, a dense set in $H \times \mathbb{C}$, we have $\langle\bar{A} Y, Z\rangle_{\bar{H}}=\left\langle Y,\left(I+\bar{b} \bar{b}^{*}\right)^{-1} Z^{\prime}\right\rangle_{\bar{H}}$. Therefore $\bar{A}^{*} Z=$ $\left(I+\bar{b} \bar{b}^{*}\right)^{-1} Z^{\prime}$ and $Z \in D\left(\bar{A}^{*}\right) \subset D(\bar{A})$.

To prove that $\widetilde{A}$ has a compact resolvent, we recall that $A$ has a compact resolvent. Moreover, the resolvent of $\bar{A}$,

$$
R_{\lambda}(\bar{A})=(\bar{A}-\lambda I)^{-1}=\left(\begin{array}{cc}
(A-\lambda I)^{-1} & 0 \\
0 & -\lambda^{-1}
\end{array}\right),
$$

is compact. We consider $(\widetilde{A}-\lambda I) R_{\lambda}(\bar{A})-I$. Using the definition of $\widetilde{A}$, we find the last operator equal to

$$
\begin{aligned}
\left(\bar{A}+\bar{b} \bar{b}^{*} \bar{A}-\lambda I\right) R_{\lambda}(\bar{A})-I & =\bar{b} \bar{b}^{*} \bar{A} R_{\lambda}(\bar{A}) \\
& =\bar{b} \bar{b}^{*}\left(I+\lambda R_{\lambda}(\bar{A})\right) .
\end{aligned}
$$

What follows is the identity

$$
\begin{aligned}
R_{\lambda}(\widetilde{A})(\widetilde{A}-\lambda I) R_{\lambda}(\bar{A}) & -R_{\lambda}(\widetilde{A}) \\
& =R_{\lambda}(\widetilde{A}) \bar{b} \bar{b}^{*}\left(I+\lambda R_{\lambda}(\bar{A})\right)
\end{aligned}
$$

and, as a consequence,

$$
R_{\lambda}(\bar{A})=R_{\lambda}(\widetilde{A})\left(I+\bar{b} \bar{b}^{*}\left(I+\lambda R_{\lambda}(\bar{A})\right)\right) .
$$

Since $R_{\lambda}(\bar{A})$ and $I+\bar{b} \bar{b}^{*}\left(I+\lambda R_{\lambda}(\bar{A})\right)$ are compact, so must be $R_{\lambda}(\widetilde{A})$.

\section{Spectral properties of the operator $\widetilde{A}$}

We shall study the spectral properties of the operator $\widetilde{A}$. First, we notice that the eigenvectors of $\bar{A}$ are $\varphi_{0}=\left(\begin{array}{l}0 \\ 1\end{array}\right)$ and $\varphi_{k}=\left(\begin{array}{c}Y_{k} \\ 0\end{array}\right)$, where $Y_{k}$ for $k>0$ are the eigenvectors of $A$.

Remark 1. It was shown by Sklyar and Szkibiel (2008b) that the eigenspaces of the operator $A$ are at most of the dimension 2. It is proved (Krabs and Sklyar, 2002) that if the parameter functions $\varrho, R, K$ and $E$ are constant (and positive) then all the eigenvalues of $A$ are simple. From now on, we assume that the eigenvalues of $A$ are simple in general. 
Utilizing the assumption from Remark 1, we write the eigenvalues of the operator $\bar{A}$ :

$$
0=\lambda_{0}<\lambda_{1}<\cdots<\lambda_{k}<\cdots \rightarrow \infty .
$$

We assume that $Y_{k}$ is an eigenvector of the operator $A$. We call the radius $r$ of the disc non-singular if

$$
\left\langle Y_{k}, b\right\rangle \neq 0
$$

for all positive integers $k$. It was shown by Sklyar and Szkibiel (2008a) that there are at most countably many singular radiuses. From know on, we consider only non-singular radiuses. We notice that Eqn. (15) holds also for the operator $\bar{A}$, i.e.,

$$
\left\langle\varphi_{k}, \bar{b}\right\rangle_{\bar{H}} \neq 0,
$$

where $\varphi_{k}, k \geq 0$, are the eigenvectors of $\bar{A}$. Indeed, for $k>0$ we have

$$
\left\langle\varphi_{k}, \bar{b}\right\rangle_{\bar{H}}=\left\langle Y_{k}, b\right\rangle \neq 0
$$

by (15) and $\left\langle\varphi_{0}, \bar{b}\right\rangle_{\bar{H}}=1 \neq 0$.

Now we are are ready to prove the main theorem about the spectrum of $\widetilde{A}$.

Theorem 2. If the eigenspaces of the operator A are onedimensional and the radius $r$ of the rotating disc is nonsingular, the following statements hold:

1. $\langle\widetilde{\varphi}, \bar{b}\rangle_{1} \neq 0$ for all eigenvectors $\widetilde{\varphi}$ of the operator $\widetilde{A}$.

2. Eigenvalues of the operator $\widetilde{A}$ are simple.

3. The eigenvalues $\widetilde{\lambda}_{k}$ of the operator $\widetilde{A}$ alternate with the ones of $\bar{A}$ :

$$
0=\lambda_{0}=\widetilde{\lambda}_{0}<\lambda_{1}<\widetilde{\lambda}_{1}<\lambda_{2}<\widetilde{\lambda}_{2}<\cdots .
$$

Proof. We first notice that $\widetilde{\varphi}_{0}=(0,0,1)^{T}$ is an eigenvector of $\widetilde{A}$ that corresponds to the eigenvalue $\widetilde{\lambda}_{0}$. Therefore, further on we consider only the eigenvalues of $\widetilde{A}$ that are different from $\widetilde{\lambda}_{0}$.

We establish the equality

$$
\left(I+\overline{b b}^{*}\right)^{-1}=I-\frac{1}{1+\|\bar{b}\|_{H}^{2}} \overline{b b}^{*} .
$$

Indeed,

$$
\begin{aligned}
\left(I+\overline{b b}^{*}\right) & \left(I-\frac{1}{1+\|\bar{b}\| \frac{2}{H}} \overline{b b}^{*}\right) \\
& =I+\overline{b b}^{*}-\frac{1}{1+\|\bar{b}\|_{\frac{2}{H}}} \overline{b b}^{*}-\frac{\|\bar{b}\|_{\frac{2}{H}}}{1+\|\bar{b}\|_{\frac{2}{H}}^{2}} \overline{b b}^{*} \\
& =I .
\end{aligned}
$$

As a consequence of (17), we obtain

$$
\left(I+\overline{b b}^{*}\right)^{-1} \bar{b}=\frac{1}{1+\|\bar{b}\| \frac{2}{H}} \bar{b} .
$$

To continue with the proof of Part 1, we take an arbitrary eigenvector $\widetilde{\varphi}$ of the operator $\widetilde{A}$ and couple it with $\bar{b}$. We have

$$
\begin{aligned}
\langle\widetilde{\varphi}, \bar{b}\rangle_{1} & =\left\langle\widetilde{\varphi},\left(I+\overline{b b}^{*}\right)^{-1} \bar{b}\right\rangle_{\bar{H}} \\
& =\frac{1}{1+\|\bar{b}\|_{\bar{H}}^{2}}\langle\widetilde{\varphi}, \bar{b}\rangle_{\bar{H}} .
\end{aligned}
$$

Thus $\langle\widetilde{\varphi}, \bar{b}\rangle_{1}=0$ if and only if $\langle\widetilde{\varphi}, \bar{b}\rangle_{\bar{H}}=0$. We shall show that if the last equality holds, then $\widetilde{\varphi}$ is an eigenvector of $\bar{A}$, but this contradicts the formula (16) and the statement 1 will be proved.

Let $\widetilde{\varphi}$ be an eigenvector of $\widetilde{A}$ with the corresponding eigenvalue $\widetilde{\lambda}$ and with $\langle\widetilde{\varphi}, \bar{b}\rangle_{\bar{H}}=0$. Using the definition of $\widetilde{A}$ and 17 we get

$$
\begin{aligned}
\bar{A} \widetilde{\varphi} & =\left(I+\overline{b b}^{*}\right)^{-1} \widetilde{A} \widetilde{\varphi} \\
& =\widetilde{\lambda}\left(I-\frac{1}{1+\|\bar{b}\|_{\frac{2}{H}}} \overline{b b}^{*}\right) \widetilde{\varphi} \\
& =\widetilde{\lambda} \widetilde{\varphi}-\frac{\widetilde{\lambda}}{1+\|\bar{b}\|_{\frac{2}{H}}} \bar{b}\langle\widetilde{\varphi}, \bar{b}\rangle_{\bar{H}} \\
& =\widetilde{\lambda} \widetilde{\varphi},
\end{aligned}
$$

which means that $\widetilde{\varphi}$ is an eigenvector of $\bar{A}$ with an eigenvalue $\tilde{\lambda}$.

To prove Part 2, we proceed again by contradiction. We assume that $\widetilde{\varphi}$ and $\widetilde{\psi}$ are two linearly independent eigenvectors of the operator $\widetilde{A}$ with one corresponding eigenvalue $\tilde{\lambda}$. Then, of course, for any $c, d \in \mathbb{C}$, $c \widetilde{\varphi}+d \widetilde{\psi}$ is also an eigenvector of $\widetilde{A}$ with the corresponding eigenvalue $\widetilde{\lambda}$. We set $c_{0}=\langle\widetilde{\psi}, \bar{b}\rangle_{1}$ and $d_{0}=-\langle\widetilde{\varphi}, \bar{b}\rangle_{1}$. Then

$$
\left\langle c_{0} \widetilde{\varphi}+d_{0} \widetilde{\psi}, \bar{b}\right\rangle_{1}=0 .
$$

This contradicts the already proved first part of the theorem.

To show the last part of the theorem, we observe that $\widetilde{\lambda}_{0}=0$ is an eigenvalue of both $\bar{A}$ and $\widetilde{A}$, but the other eigenvalues of $\bar{A}$ are not the eigenvalues of the operator $\widetilde{A}$. Indeed, we assume that $\lambda \neq 0$ is a common eigenvalue, i.e., $\lambda \widetilde{\varphi}=\widetilde{A} \widetilde{\varphi}$ and $\lambda \varphi=\bar{A} \varphi$, where $\widetilde{\varphi}$ is an eigenvector of $\widetilde{A}, \varphi$, an eigenvector of $\bar{A}$ and both of them correspond to $\lambda$. Then we have

$$
\lambda \widetilde{\varphi}=\left(I+\overline{b b}^{*}\right) \bar{A} \widetilde{\varphi}=\bar{A} \widetilde{\varphi}+\bar{b}\langle\bar{A} \widetilde{\varphi}, \bar{b}\rangle_{\bar{H}} .
$$


A consequence of the above equation is the following:

$$
\lambda\langle\widetilde{\varphi}, \varphi\rangle_{\bar{H}}=\langle\bar{A} \widetilde{\varphi}, \varphi\rangle_{\bar{H}}+\langle\bar{A} \widetilde{\varphi}, \bar{b}\rangle_{\bar{H}}\langle\bar{b}, \varphi\rangle_{\bar{H}} .
$$

We recall that the eigenvector $\varphi$ of $\bar{A}$ must be of the form $\left(\begin{array}{c}Y \\ 0\end{array}\right)$, where $Y$ is the eigenvector of the operator $A$. Therefore the corresponding eigenvalue $\lambda$ is a positive real number. Hence we get

$$
\langle\bar{A} \widetilde{\varphi}, \varphi\rangle_{\bar{H}}=\langle\widetilde{\varphi}, \bar{A} \varphi\rangle_{\bar{H}}=\lambda\langle\widetilde{\varphi}, \varphi\rangle_{\bar{H}}
$$

Combining the above two equations, we obtain

$$
\langle\bar{A} \widetilde{\varphi}, \bar{b}\rangle_{\bar{H}}\langle\bar{b}, \varphi\rangle_{\bar{H}}=0
$$

that holds if and only if $\langle\bar{b}, \varphi\rangle_{\bar{H}}=0$ or $\langle\bar{A} \widetilde{\varphi}, \bar{b}\rangle_{\bar{H}}=0$. The first equation is impossible by (16), while the other one implies, together with (18), that $\widetilde{\varphi}$ is an eigenvector of $\bar{A}$ with the corresponding eigenvalue $\lambda$. But then $\langle\bar{A} \widetilde{\varphi}, \bar{b}\rangle_{\bar{H}}=\lambda\langle\widetilde{\varphi}, \bar{b}\rangle_{\bar{H}}=0$, which is impossible by the already proved Part 1.

Proceeding further, let $\lambda$ be an eigenvalue of $\widetilde{A}$ with the corresponding eigenvector $\widetilde{\varphi}$. Then

$$
\bar{A} \widetilde{\varphi}=\left(I+\overline{b b}^{*}\right)^{-1} \widetilde{A} \widetilde{\varphi}=\lambda\left(I+\overline{b b}^{*}\right)^{-1} \widetilde{\varphi} .
$$

By (17) it follows that

$$
\bar{A} \widetilde{\varphi}=\lambda\left(I-\frac{\overline{b b}^{*}}{1+\|\bar{b}\|_{\frac{2}{H}}^{2}}\right) \widetilde{\varphi},
$$

and after some elementary transformations we obtain

$$
(\bar{A}-\lambda I) \widetilde{\varphi}=-\frac{\lambda\langle\widetilde{\varphi}, \bar{b}\rangle_{\bar{H}}}{1+\|\bar{b}\|_{\bar{H}}^{2}} .
$$

The above equation is equivalent to

$$
\widetilde{\varphi}=-\frac{\lambda\langle\widetilde{\varphi}, \bar{b}\rangle_{\bar{H}}}{1+\|\bar{b}\|_{\bar{H}}^{2}} R_{\lambda}(\bar{A}) \bar{b}
$$

Therefore $R_{\lambda}(\bar{A}) \bar{b}$ is an eigenvector of $\widetilde{A}$ corresponding to the eigenvalue $\lambda$ and as such must satisfy [19). Hence

$$
R_{\lambda}(\bar{A}) \bar{b}=-\frac{\lambda\left\langle R_{\lambda}(\bar{A}) \bar{b}, \bar{b}\right\rangle_{\bar{H}}}{1+\|\bar{b}\|_{\bar{H}}^{2}} R_{\lambda}(\bar{A}) \bar{b}
$$

or

$$
\left(1+\frac{\lambda\left\langle R_{\lambda}(\bar{A}) \bar{b}, \bar{b}\right\rangle_{\bar{H}}}{1+\|\bar{b}\|_{\bar{H}}^{2}}\right) R_{\lambda}(\bar{A}) \bar{b}=0 .
$$

What follows is

$$
\left(1+\frac{\lambda\left\langle R_{\lambda}(\bar{A}) \bar{b}, \bar{b}\right\rangle_{\bar{H}}}{1+\|\bar{b}\|_{\bar{H}}^{2}}\right)=0 .
$$

We consider the identity $\lambda R_{\lambda}(\bar{A})=-I+\bar{A} R_{\lambda}(\bar{A})$. Applying it to 20, we get

$$
\frac{1+\left\langle\bar{A} R_{\lambda}(\bar{A}) \bar{b}, \bar{b}\right\rangle_{\bar{H}}}{1+\|\bar{b}\|_{\bar{H}}^{2}}=0 .
$$

Therefore the equation

$$
\left\langle\bar{A} R_{\lambda}(\bar{A}) \bar{b}, \bar{b}\right\rangle_{\bar{H}}=-1
$$

must be satisfied by all non-zero eigenvalues $\lambda$ of $\widetilde{A}$.

Let $\left(\varphi_{n}\right)$ be the (orthonormal) sequence of all eigenvectors of $\bar{A}$ with $\lambda_{n}$ being the eigenvalue corresponding to $\varphi_{n}$ ( $n$ is a non-negative integer). Then, for some coefficients $b_{n}$,

$$
\bar{b}=\sum_{n=0}^{\infty} b_{n} \varphi_{n}, \quad \bar{A} R_{\lambda}(\bar{A}) \bar{b}=\sum_{n=1}^{\infty} \frac{\lambda_{n}}{\lambda_{n}-\lambda} b_{n} \varphi_{n} .
$$

Moreover, the series $\sum_{n=1}^{\infty}\left|b_{n}\right|^{2}$ converges and (21) takes form

$$
\sum_{n=1}^{\infty} \frac{\lambda_{n}}{\lambda_{n}-\lambda}\left|b_{n}\right|^{2}=-1 .
$$

We define

$$
f(x)=\sum_{n=1}^{\infty} \frac{\lambda_{n}}{\lambda_{n}-x}\left|b_{n}\right|^{2}+1
$$

for all $x \neq \lambda_{n}$, where $n$ are positive integers. We notice that, due to (22), the zeroes of $f$ are exactly at the eigenvalues of $\widetilde{A}$. For those values of $x$ for which $f$ is defined,

$$
f^{\prime}(x)=\sum_{n=1}^{\infty} \frac{\lambda_{n}}{\left(\lambda_{n}-x\right)^{2}}\left|b_{n}\right|^{2} .
$$

Since $f^{\prime}(x)>0$, the function $f$ strictly increases on each interval $\left(\lambda_{n}, \lambda_{n+1}\right)$. Moreover, for each positive integer $n$

$$
\lim _{x \rightarrow \lambda_{n}^{-}} f(x)=+\infty, \quad \lim _{x \rightarrow \lambda_{n}^{+}} f(x)=-\infty .
$$

Thus $f$ has exactly one zero in $\left(\lambda_{n}, \lambda_{n+1}\right)$. Furthermore, since $\lim _{x \rightarrow-\infty} f(x)=0$ and the function $f$ increases, it has no zeroes on $\left(-\infty, \lambda_{1}\right)$. Hence Part 3 is true.

Corollary 1. The eigenvectors of $\widetilde{A}$ form an orthonormal basis in $\bar{H}$ with the inner product $\langle\cdot, \cdot\rangle_{1}$.

\section{Weak solution}

As has been noticed (Theorem 2), the operator $\widetilde{A}$ has simple and non-negative eigenvalues. Therefore, we may define $\widetilde{A}^{1 / 2}: D\left(\widetilde{A}^{1 / 2}\right) \rightarrow \bar{H}$ by the formula

$$
\widetilde{A}^{1 / 2} v=\sum_{k=0}^{\infty} \sqrt{\tilde{\lambda}_{k}}\left\langle v, \widetilde{\varphi}_{k}\right\rangle \widetilde{\varphi}_{k},
$$


where $\left(\widetilde{\varphi}_{k}\right)_{k \geq 0}$ is the (normalized) sequence of eigenvectors of the operator $\widetilde{A}$ with $\left(\tilde{\lambda}_{k}\right)_{k \geq 0}$ being the sequence of the corresponding eigenvalues and

$$
D\left(\widetilde{A}^{1 / 2}\right)=\left\{v \in \bar{H}: \sum_{k=0}^{\infty} \tilde{\lambda}_{k}\left|\left\langle v, \widetilde{\varphi}_{k}\right\rangle\right|^{2}<\infty\right\} .
$$

We adjust the inner product $\langle\cdot, \cdot\rangle_{1}$ in $\bar{H}$ by defining for $v, w \in D\left(\widetilde{A}^{1 / 2}\right)$ the function

$$
\langle v, w\rangle_{2}=\left\langle v, \widetilde{\varphi}_{0}\right\rangle_{1}\left\langle\widetilde{\varphi}_{0}, w\right\rangle_{1}+\sum_{k=1}^{\infty} \tilde{\lambda}_{k}\left\langle v, \widetilde{\varphi}_{k}\right\rangle_{1}\left\langle\widetilde{\varphi}_{k}, w\right\rangle_{1}
$$

With the inner product $\langle\cdot, \cdot\rangle_{2}$, the set $D\left(\widetilde{A}^{1 / 2}\right)$ becomes a Hilbert space with the norm $\|\cdot\|_{2}$.

There is exactly one weak solution of Eqn. (12) with the initial conditions

$$
\begin{aligned}
w(x, 0) & =\dot{w}(x, 0)=0, \\
\xi(x, 0) & =\dot{\xi}(x, 0)=0, \\
\theta(0) & =\dot{\theta}(0)=0
\end{aligned}
$$

for $x \in[0,1]$ and $T>0$, which consist of the continuous functions

$$
\left(\begin{array}{c}
w(x, \cdot) \\
\xi(x, \cdot) \\
\theta(\cdot)
\end{array}\right):[0, T] \rightarrow D\left(\widetilde{A}^{1 / 2}\right)
$$

and, simultaneously, those functions are of class $C^{1}$ from $[0, T]$ to $\bar{H}$. It is given by the following formula:

$$
\begin{aligned}
\left(\begin{array}{c}
w(x, t) \\
\xi(x, t) \\
\theta(t)
\end{array}\right)= & \left(\int_{0}^{t}(t-s)\left\langle\bar{b}, \widetilde{\varphi}_{0}\right\rangle_{1} \widetilde{u}(s) \mathrm{d} s\right) \widetilde{\varphi}_{0}(x) \\
& +\sum_{k=1}^{\infty} \frac{1}{\sqrt{\widetilde{\lambda}_{k}}} S_{k}(t) \widetilde{\varphi}_{k}(x)
\end{aligned}
$$

where

$$
S_{k}(t)=\int_{0}^{t} \sin \left(\sqrt{\widetilde{\lambda}_{k}}(t-s)\right)\left\langle\bar{b}, \widetilde{\varphi}_{k}\right\rangle_{1} \widetilde{u}(s) \mathrm{d} s .
$$

Its (time) derivative is given by

$$
\left(\begin{array}{c}
\dot{w}(x, t) \\
\dot{\xi}(x, t) \\
\dot{\theta}(t)
\end{array}\right)=\sum_{k=0}^{\infty} \dot{S}_{k}(t) \widetilde{\varphi}_{k}(x)
$$

where

$$
\dot{S}_{k}(t)=\int_{0}^{t} \cos \left(\sqrt{\widetilde{\lambda}_{k}}(t-s)\right)\left\langle\bar{b}, \widetilde{\varphi}_{k}\right\rangle_{1} \widetilde{u}(s) \mathrm{d} s .
$$

In the next theorem, we shall show that the solution (23) is, in fact, a member of $D\left(A^{1 / 2}\right) \times \mathbb{C}$.
Theorem 3. The domains of the operators $\widetilde{A}^{1 / 2}$ and $\bar{A}^{1 / 2}$ are equal to

$$
\left\{v \in \bar{H}: \sum_{j=0}^{\infty} \lambda_{j}\left|\left\langle v, \varphi_{j}\right\rangle_{H}\right|^{2}<\infty\right\}
$$

This is, in turn, equal to $D\left(A^{1 / 2}\right) \times \mathbb{C}$.

Proof. It is obvious that the set is equal to $D\left(A^{1 / 2}\right) \times \mathbb{C}$ and to $D\left(\bar{A}^{1 / 2}\right)$. Let us write the norms in both $D\left(\widetilde{A}^{1 / 2}\right)$ and $D\left(A^{1 / 2}\right) \times \mathbb{C}$ :

$$
\begin{aligned}
& \|v\|_{2}=\left(\left|\left\langle v, \widetilde{\varphi}_{0}\right\rangle_{1}\right|^{2}+\sum_{k=1}^{\infty} \widetilde{\lambda}_{k}\left|\left\langle v, \widetilde{\varphi}_{k}\right\rangle_{1}\right|^{2}\right)^{1 / 2}, \\
& \|v\|_{3}=\left(\left|\left\langle v, \varphi_{0}\right\rangle_{\bar{H}}\right|^{2}+\sum_{k=1}^{\infty} \lambda_{k}\left|\left\langle v, \varphi_{k}\right\rangle_{\bar{H}}\right|^{2}\right)^{1 / 2} .
\end{aligned}
$$

In the following lemma, we shall prove that on $D(A) \times \mathbb{C}$ the norms $\|\cdot\|_{2}$ and $\|\cdot\|_{3}$ are equivalent.

Lemma 1. Let $\left(v_{n}\right)$ be a sequence in $D(A) \times \mathbb{C}$. Then $\left(v_{n}\right)$ converges in $\|\cdot\|_{2}$ if and only if it converges in the norm $\|\cdot\|_{3}$. Moreover, if $\left\|v_{n}-v\right\|_{2} \rightarrow 0$ and $\left\|v_{n}-v^{\prime}\right\|_{3} \rightarrow 0$, for some $v$ and $v^{\prime}$, then $v=v^{\prime}$.

Proof. First, we assume that $\left(v_{n}\right)$ is convergent to some $v$ in the norm $\|\cdot\|_{2}$. Then, according to the norm definition,

$$
\begin{aligned}
\left\|v_{n}-v_{m}\right\|_{2}^{2}= & \left|\left\langle v_{n}-v_{m}, \widetilde{\varphi}_{0}\right\rangle_{1}\right|^{2} \\
& +\sum_{k=1}^{\infty} \widetilde{\lambda}_{k}\left|\left\langle v_{n}-v_{m}, \widetilde{\varphi}_{k}\right\rangle_{1}\right|^{2}
\end{aligned}
$$

for any positive integers $n$ and $m$. But, for all $k \geq 1$,

$$
\begin{aligned}
\tilde{\lambda}_{k}\left|\left\langle v_{n}-v_{m}, \widetilde{\varphi}_{k}\right\rangle_{1}\right|^{2} & \\
= & \left\langle v_{n}-v_{m}, \widetilde{\lambda}_{k} \widetilde{\varphi}_{k}\right\rangle_{1}\left\langle\widetilde{\varphi}_{k}, v_{n}-v_{m}\right\rangle_{1} \\
& =\left\langle\widetilde{A}\left(v_{n}-v_{m}\right), \widetilde{\varphi}_{k}\right\rangle_{1}\left\langle\widetilde{\varphi}_{k}, v_{n}-v_{m}\right\rangle_{1},
\end{aligned}
$$

because $v_{n}$ and $v_{m}$ belong to $D(A) \times \mathbb{C}=D(\bar{A})$ and the operator $\widetilde{A}$ is self-adjoint. We remark that, if $\left(\varphi_{k}\right)$ forms an orthonormal basis in a Hilbert space and $v, w$ are some elements in this space, then

$$
\sum_{k=1}^{\infty}\left\langle v, \varphi_{k}\right\rangle\left\langle\varphi_{k}, w\right\rangle=\langle v, w\rangle .
$$

Accordingly we have the following continuation of the reasoning started in 27):

$$
\begin{aligned}
\| v_{n} & -v_{m} \|_{2}^{2} \\
& =\left|\left\langle v_{n}-v_{m}, \widetilde{\varphi}_{0}\right\rangle_{1}\right|^{2}+\left\langle\widetilde{A}\left(v_{n}-v_{m}\right), v_{n}-v_{m}\right\rangle_{1} \\
& =\left|\left\langle v_{n}-v_{m}, \widetilde{\varphi}_{0}\right\rangle_{1}\right|^{2}+\left\langle\bar{A}\left(v_{n}-v_{m}\right), v_{n}-v_{m}\right\rangle_{\bar{H}} .
\end{aligned}
$$


Therefore if $\left\|v_{n}-v_{m}\right\|_{2}^{2} \rightarrow 0$ as $n, m \rightarrow \infty$, so does

$$
\left\langle\bar{A}\left(v_{n}-v_{m}\right), v_{n}-v_{m}\right\rangle_{\bar{H}} .
$$

On the other hand,

$$
\|v\|_{1}^{2} \leq \sum_{k=0}^{\infty}\left|\left\langle v, \widetilde{\varphi}_{k}\right\rangle_{1}\right|^{2} \leq\|v\|_{2}^{2}
$$

for any $v$. Therefore $\left\|v_{n}-v_{m}\right\|_{1} \rightarrow 0$ as $n, m \rightarrow \infty$ if $\left\|v_{n}-v_{m}\right\|_{2} \rightarrow 0$. Due to the equivalence of the norms $\|\cdot\|_{1}$ and $\|\cdot\|_{\bar{H}}$, we also have $\left\|v_{n}-v_{m}\right\|_{\bar{H}} \rightarrow 0$.

Similarly we arrive at the following formula:

$$
\begin{aligned}
& \left\|v_{n}-v_{m}\right\|_{3}^{2} \\
& \quad=\left|\left\langle v_{n}-v_{m}, \varphi_{0}\right\rangle_{\bar{H}}\right|^{2}+\left\langle\bar{A}\left(v_{n}-v_{m}\right), v_{n}-v_{m}\right\rangle_{\bar{H}},
\end{aligned}
$$

and what follows is the inequality

$$
\begin{aligned}
& \left\|v_{n}-v_{m}\right\|_{3}^{2} \\
& \quad \leq\left\|v_{n}-v_{m}\right\|_{H}^{2}+\left\langle\bar{A}\left(v_{n}-v_{m}\right), v_{n}-v_{m}\right\rangle_{\bar{H}} .
\end{aligned}
$$

Therefore, we finally obtain that for $n, m \rightarrow \infty$ we have $\left\|v_{n}-v_{m}\right\|_{3} \rightarrow 0$ if $\left\|v_{n}-v_{m}\right\|_{2} \rightarrow 0$. Thus $\left(v_{n}\right)$ is a Cauchy sequence in the norm $\|\cdot\|_{3}$, so it converges to some $v^{\prime}$.

\section{Since}

$$
\left\|v_{n}-v\right\|_{2} \geq\left\|v_{n}-v\right\|_{1}, \quad\left\|v_{n}-v^{\prime}\right\|_{3} \geq\left\|v_{n}-v^{\prime}\right\|_{\bar{H}}
$$

and the norms $\|\cdot\|_{1},\|\cdot\|_{\bar{H}}$ are equivalent, we have

$$
\left\|v_{n}-v\right\|_{1} \rightarrow 0 \quad \text { and } \quad\left\|v_{n}-v^{\prime}\right\|_{\bar{H}} \rightarrow 0
$$

as $n$ tends to infinity. Therefore, $v=v^{\prime}$.

The inverse assertion can be proved with the use of a similar argument.

As $D\left(\widetilde{A}^{1 / 2}\right)$ and $D\left(A^{1 / 2}\right) \times \mathbb{C}$ are the completions of $D(\bar{A})$ and $D(A) \times \mathbb{C}$, respectively, and the last two are equal, it follows from the above lemma that $D\left(\widetilde{A}^{1 / 2}\right)=$ $D\left(A^{1 / 2}\right) \times \mathbb{C}$. To show the equivalence of the norms on the completions, we consider the identity

$$
I:\left(D\left(\widetilde{A}^{1 / 2}\right),\|\cdot\|_{2}\right) \rightarrow\left(D\left(A^{1 / 2}\right) \times \mathbb{C},\|\cdot\|_{3}\right) .
$$

By Lemma 1, $I$ is continuous at 0 , so it is bounded. The inverse $I^{-1}$ is also bounded, so $\|\cdot\|_{2}$ and $\|\cdot\|_{3}$ are equivalent.

\section{Approximate control}

Let us write the final state of the object considered:

$$
Y_{T}=\left(\begin{array}{c}
w(\cdot, T) \\
\xi(\cdot, T) \\
\theta(T)
\end{array}\right) \quad \dot{Y}_{T}=\left(\begin{array}{c}
\dot{w}(\cdot, T) \\
\dot{\xi}(\cdot, T) \\
\dot{\theta}(T)
\end{array}\right)
$$

for $T>0$, and define the operator

$$
\widetilde{S}: L^{2}(0, T) \rightarrow\left(D\left(A^{1 / 2}\right) \times \mathbb{C}\right) \times \bar{H}
$$

by the formula

$$
\widetilde{S}(v)=\left(\begin{array}{l}
\sum_{k=0}^{\infty}\left(\int_{0}^{T} S_{k}(t) v(t) \mathrm{d} t\right) \widetilde{\varphi}_{k} \\
\sum_{k=0}^{\infty}\left(\int_{0}^{T} \dot{S}_{k}(t) v(t) \mathrm{d} t\right) \widetilde{\varphi}_{k}
\end{array}\right) .
$$

Here

for $k>0$ and

$$
\begin{aligned}
& S_{0}(t)=a_{0}(T-t) \\
& S_{k}(t)=\frac{1}{\sqrt{\tilde{\lambda}_{k}}} a_{k} \sin \sqrt{\widetilde{\lambda}_{k}}(T-t)
\end{aligned}
$$

$$
\begin{aligned}
\dot{S}_{k}(t) & =a_{k} \cos \sqrt{\widetilde{\lambda}_{k}}(T-t), \\
a_{k} & =\left\langle\bar{b}, \widetilde{\varphi}_{k}\right\rangle_{1}
\end{aligned}
$$

for $k \geq 0$. We notice that the operator $\widetilde{S}$ is continuous. Also, we see that if $\widetilde{u}$ is a control, then $\widetilde{S}(\widetilde{u})$ is the final state (compare with the weak solution (24), (25)). Therefore the one of approximate controllability becomes the problem of proving that the range of the operator $\widetilde{S}$ is dense in $\left.D\left(A^{1 / 2}\right) \times \mathbb{C}\right) \times \bar{H}$. This, however, is the problem of proving that the adjoint operator $\widetilde{S}^{*}$ is injective.

Theorem 4. Assuming the disc radius is non-singular and

$$
T>T_{0}=2\left(\int_{0}^{1} \sqrt{\frac{\varrho(x)}{K(x)}} d x+\int_{0}^{1} \sqrt{\frac{R(x)}{E(x)}} d x\right),
$$

the system (1)-(2) is approximately controllable from the position of rest.

Proof. We have in explicit form

$$
\begin{aligned}
\widetilde{S}^{*}:( & \left.\left(A^{1 / 2}\right) \times \mathbb{C}\right) \times \bar{H} \rightarrow L^{2}(0, T), \\
\widetilde{S}^{*}\left(\begin{array}{c}
Y \\
Z
\end{array}\right)(t)= & \left\langle Y, \widetilde{\varphi}_{0}\right\rangle_{1} a_{0}(T-t) \\
& +\sum_{k=1}^{\infty}\left\langle Y, \widetilde{\varphi}_{k}\right\rangle_{1} \frac{a_{k}}{\sqrt{\widetilde{\lambda}_{k}}} \sin \sqrt{\widetilde{\lambda}_{k}}(T-t) \\
& +\sum_{k=0}^{\infty}\left\langle Z, \widetilde{\varphi}_{k}\right\rangle_{1} a_{k} \cos \sqrt{\widetilde{\lambda}_{k}}(T-t)
\end{aligned}
$$

for almost all $t \in[0, T]$. Because the radius is non-singular, $a_{k} \neq 0$ for all non-negative values of $k$. 
Let

$$
\begin{aligned}
& d(x)=\max \left\{k: \sqrt{\lambda_{k}}<x\right\}, \\
& \widetilde{d}(x)=\max \left\{k: \sqrt{\widetilde{\lambda}_{k}}<x\right\} .
\end{aligned}
$$

From Theorem 2$] d(x)-1<\tilde{d}(x)<d(x)$. Therefore

$$
\limsup _{x, y \rightarrow \infty} \frac{\tilde{d}(x+y)-\tilde{d}(x)}{y}=\limsup _{x, y \rightarrow \infty} \frac{d(x+y)-d(x)}{y} .
$$

By the classical theorem of Paley and Wiener (1934) in the version presented by Russell (1967) and reasoning similar to the one of Sklyar and Szkibiel (2008b), we obtain that the limit 28 is less than $T / 2 \pi$ provided $T>T_{0}$. This means that the system

$$
\left\{1, t, \sin \sqrt{\tilde{\lambda}_{k}} t, \cos \sqrt{\widetilde{\lambda}_{k}} t: k>0\right\}
$$

is minimal for $T>T_{0}$. In particular, the equality $\widetilde{S}^{*}\left(\begin{array}{c}Y \\ Z\end{array}\right)=0$ holds only if $\left\langle Y, \widetilde{\varphi}_{k}\right\rangle_{1}=\left\langle Z, \widetilde{\varphi}_{k}\right\rangle_{1}=0$ for $k \geq 0$. Since the system $\left(\widetilde{\varphi}_{k}\right)$ is complete, we obtain $Y=Z=0$. Therefore $\widetilde{S}^{*}$ is injective and, as remarked above, the system is approximately controllable.

\section{Stabilizability}

Gathering together the pieces of our discussion, we need to find the bounded linear functional $p=p(Y, \dot{Y})$, such that every solution $Y(t)=(w(\cdot, t), \xi(\cdot, t), \theta(t))$ of Eqn. (14) with the feedback control

$$
\tilde{u}(t)=p(w(\cdot, t), \xi(\cdot, t), \theta(t), \dot{w}(\cdot, t), \dot{\xi}(\cdot, t), \dot{\theta}(t))
$$

tends to 0 as $t \rightarrow \infty$ in the sense of extinguishing the total energy of the system, i.e., (6) is satisfied.

To deal with this problem, we need to rearrange the operators and functionals we have dealt with before. Thus we define $\bar{b}^{+}: \bar{H} \rightarrow \mathbb{C}$ by the formula

$$
\bar{b}^{+} Y=\langle Y, \bar{b}\rangle_{1} .
$$

Then we define the control $\tilde{u}_{0}=\tilde{u}+\bar{b}^{+} Y$ so Eqn. (14) takes the form

$$
\ddot{Y}+A_{0} Y=\bar{b} \tilde{u}_{0}
$$

with $A_{0}=\widetilde{A}+\bar{b} \bar{b}^{+}$defined on $D(\bar{A})$.

Proposition 1. The operator $A_{0}$ is self adjoint (with respect to $\left.\langle\cdot, \cdot\rangle_{1}\right)$ and has a compact resolvent.

For the proof, we notice that $A_{0}$ is a finite dimensional perturbation of a self-adjoint operator $\widetilde{A}$ with compact resolvent (Kato, 1966).

Moreover, one can easily see that $\left\langle A_{0} Y, Y\right\rangle_{1} \geq 0$ and the equality means $\widetilde{A} Y=0$ and finally $\langle Y, \bar{b}\rangle_{1}=0$, which contradicts Part 1 of Theorem 2 or Theorem 1
Thus the operator $A_{0}$ has a discrete spectrum with (strictly) positive eigenvalues. Using the arguments from the proof of Part 3 of Theorem 2, we can prove that the eigenvalues of $A_{0}$ are simple. What follows is the possessing of a complete orthonormal sequence $\left\{\psi_{k}\right\}_{k \in \mathbb{N}_{0}}$ of eigenvectors that correspond to a sequence $\left\{\mu_{k}\right\}_{k \in \mathbb{N}_{0}}$ of simple positive eigenvalues $\left(\mathbb{N}_{0}\right.$ denotes the set of non-negative integers).

We shall use the operator $A_{0}^{1 / 2}: D\left(A_{0}^{1 / 2}\right) \rightarrow \bar{H}$ given by the formulas

$$
A_{0}^{1 / 2} v=\sum_{j=0}^{\infty} \sqrt{\mu_{j}}\left\langle v, \psi_{j}\right\rangle_{1} \psi_{j}
$$

and

$$
D\left(A_{0}^{1 / 2}\right)=\left\{v \in \bar{H}: \sum_{j=0}^{\infty} \mu_{j}\left|\left\langle v, \psi_{j}\right\rangle_{1}\right|<\infty\right\} .
$$

The latter is a Hilbert space with the inner product

$$
\langle v, w\rangle_{4}=\sum_{j=0}^{\infty} \mu_{j}\left\langle v, \psi_{j}\right\rangle_{1}\langle\psi, w\rangle_{1} .
$$

Lemma 2. We assume that $\left(v_{n}\right)$ is a sequence of elements from $D(A) \times \mathbb{C}$. Then $\left(v_{n}\right)$ is convergent in the norm $\|\cdot\|_{4}$ (generated by the inner product $\left.\langle\cdot, \cdot\rangle_{4}\right)$ if and only if it is convergent in the norm $\|\cdot\|_{2}$. Moreover, if $\left\|v_{n}-v\right\|_{4} \rightarrow 0$ for some $v$ and $\left\|v_{n}-v^{\prime}\right\|_{2} \rightarrow 0$ for some $v^{\prime}$ (as $\left.n \rightarrow \infty\right)$, then $v=v^{\prime}$.

Proof. We assume that

$$
\lim _{n \rightarrow \infty} v_{n}=v
$$

for some $v \in D\left(A_{0}^{1 / 2}\right)$. Then $\left(v_{n}\right)$ is a Cauchy sequence in $\|\cdot\|_{4}$. Since $v_{n} \in D(\bar{A})$ for all positive integers $n$, we have for $n, m \in \mathbb{N}$

$$
\begin{aligned}
\left\|v_{n}-v_{m}\right\|_{4}^{2}= & \left\langle A_{0}\left(v_{n}-v_{m}\right), v_{n}-v_{m}\right\rangle_{1} \\
= & \left\langle\widetilde{A}\left(v_{n}-v_{m}\right), v_{n}-v_{m}\right\rangle_{1} \\
& +\left|\left\langle\bar{b}, v_{n}-v_{m}\right\rangle_{1}\right|^{2}
\end{aligned}
$$

Since $\left\|v_{n}-v_{m}\right\|_{4} \rightarrow 0$ as $n, m \rightarrow \infty$, we have

$$
\left\langle\widetilde{A}\left(v_{n}-v_{m}\right), v_{n}-v_{m}\right\rangle_{1} \rightarrow 0
$$

and

$$
\left\|\left\langle\bar{b}, \widetilde{\varphi}_{0}\right\rangle_{1}\left\langle\widetilde{\varphi}_{0}, v_{n}-v_{m}\right\rangle_{1}+\sum_{j=1}^{\infty}\left\langle\bar{b}, \widetilde{\varphi}_{j}\right\rangle_{1}\left\langle\widetilde{\varphi}_{j}, v_{n}-v_{m}\right\rangle_{1}\right\|
$$

equal to $\left|\left\langle\bar{b}, v_{n}-v_{m}\right\rangle_{1}\right|$ tends to 0 .

Further on, we notice that

$$
\left\langle\widetilde{A}\left(v_{n}-v_{m}\right), v_{n}-v_{m}\right\rangle_{1} \geq \lambda_{1} \sum_{j=1}^{\infty}\left|\left\langle\widetilde{\varphi}_{j}, v_{n}-v_{m}\right\rangle_{1}\right|^{2} .
$$


Hence, by the Hölder inequality and (30),

$$
\begin{aligned}
& \left\|\sum_{j=1}^{\infty}\left\langle\bar{b}, \widetilde{\varphi}_{j}\right\rangle_{1}\left\langle\widetilde{\varphi}_{j}, v_{n}-v_{m}\right\rangle_{1}\right\|^{2} \\
& \quad \leq \sum_{j=1}^{\infty}\left|\left\langle\bar{b}, \widetilde{\varphi}_{j}\right\rangle_{1}\right|^{2} \sum_{j=1}^{\infty}\left|\left\langle\widetilde{\varphi}_{j}, v_{n}-v_{m}\right\rangle_{1}\right|^{2} \rightarrow 0
\end{aligned}
$$

as $n, m \rightarrow \infty$. In addition to that $\left\langle\bar{b}, \widetilde{\varphi}_{0}\right\rangle_{1} \neq 0$, so $\left\langle\widetilde{\varphi}_{0}, v_{n}-v_{m}\right\rangle_{1} \rightarrow 0$. Finally, as $n, m \rightarrow \infty$,

$$
\begin{aligned}
& \left\|v_{n}-v_{m}\right\|_{2}^{2} \\
& =\left|\left\langle v_{n}-v_{m}, \widetilde{\varphi}_{0}\right\rangle_{1}\right|^{2}+\left\langle\widetilde{A}\left(v_{n}-v_{m}\right), v_{n}-v_{m}\right\rangle_{1} \\
& \quad \rightarrow 0 .
\end{aligned}
$$

This means that $\left(v_{n}\right)$ is a Cauchy sequence in $\|\cdot\|_{2}$, so it must be convergent to some $v^{\prime} \in D\left(\widetilde{A}^{1 / 2}\right)$. In order to finish the proof, we need to see that $v=v^{\prime}$. We notice that $\left\|v_{n}-v_{m}\right\|_{2} \geq\left\|v_{n}-v^{\prime}\right\|_{1}$ and $\left\|v_{n}-v_{m}\right\|_{4} \geq \sqrt{\mu_{0}} \| v_{n}-$ $v \|_{1}$. Therefore, both $\left\|v_{n}-v^{\prime}\right\|_{1}$ and $\left\|v_{n}-v\right\|_{1}$ tend to 0 as $n \rightarrow \infty$. But in $\|\cdot\|_{1}$ the limit is unique, so $v=v^{\prime}$.

We proceed in a similar way to prove the inverse assertion.

Corollary 2. The following holds:

(i) $D\left(A_{0}^{1 / 2}\right)=D\left(\widetilde{A}^{1 / 2}\right)=D\left(A^{1 / 2}\right) \times \mathbb{C}$,

(ii) $\|\cdot\|_{2},\|\cdot\|_{3}$ and $\|\cdot\|_{4}$ are equivalent.

The proof follows directly from the above lemma and Lemma 1

We conclude this section with the main result on stabilizability (Theorem 6). In the proof we shall use the following theorem, due to Krabs and Sklyar (2002) as well as Sklyar and Rezounenko (2003).

Theorem 5. Let $\sigma\left(\mathcal{A}_{0}\right) \cap i \mathbb{R}$ be at most countable and $\mathcal{A}_{0}$ generate a contractive $C^{0}$-semigroup. Then Eqn. (31) is strongly stabilizable if and only if no eigenvector $\psi$ of the operator $\mathcal{A}_{0}$ corresponding to an imaginary eigenvalue satisfies $\left\langle b_{0}, \psi\right\rangle_{\mathcal{H}}=0$. The stabilizing control is then given by $\tilde{u}_{0}=-\left\langle Z, b_{0}\right\rangle_{\mathcal{H}}$.

Theorem 6. Assume the radius of the disk is non-singular. The system (14) is strongly stabilizable with the control

$$
\tilde{u}=-\left\langle Y+\dot{Y},\left(I+\bar{b} \bar{b}^{*}\right)^{-1} \bar{b}\right\rangle_{\bar{H}} .
$$

Proof. We shall first set up a system of equations of the first order. Let $Z:[0, T] \rightarrow D(\bar{A}) \times \bar{H}$ be given by the equation

$$
Z(t)=\left(\begin{array}{c}
Y(t) \\
\dot{Y}(t)
\end{array}\right) \text { fort } \in[0, T] .
$$

Then we define the operator $\mathcal{A}_{0}: D(\bar{A}) \times \bar{H} \rightarrow \bar{H} \times \bar{H}$ by the formula

$$
\mathcal{A}_{0}\left(\begin{array}{c}
Y_{1} \\
Y_{2}
\end{array}\right)=\left(\begin{array}{c}
Y_{2} \\
-A_{0} Y_{1}
\end{array}\right)
$$

In addition to this, let

$$
b_{0}=\left(\begin{array}{l}
0 \\
\bar{b}
\end{array}\right)
$$

Then Eqn. 29) takes the form

$$
\dot{Z}=\mathcal{A}_{0} Z+b_{0} \tilde{u}_{0}
$$

Now, we utilize the Hilbert space $\mathcal{H}=D\left(A_{0}^{1 / 2}\right) \times \bar{H}$ with the inner product defined as follows:

$$
\left\langle Z_{1}, Z_{2}\right\rangle_{\mathcal{H}}=\left\langle V_{1}, V_{2}\right\rangle_{4}+\left\langle W_{1}, W_{2}\right\rangle_{1},
$$

where

$$
Z_{1}=\left(\begin{array}{c}
V_{1} \\
W_{1}
\end{array}\right), \quad Z_{2}=\left(\begin{array}{c}
V_{2} \\
W_{2}
\end{array}\right)
$$

$Z_{1}, Z_{2} \in \mathcal{H}$. Then $\mathcal{A}_{0}: D(\bar{A}) \times D\left(A_{0}^{1 / 2}\right) \rightarrow \mathcal{H}$ generates a contractive $C^{0}$-semigroup on $\mathcal{H}$. Moreover, $\mathcal{A}_{0}$ has a discrete spectrum $\left\{\mu_{k}^{+}, \mu_{k}^{-}: k \in \mathbb{N}_{0}\right\}$ given by

$$
\mu_{k}^{+}=i \sqrt{\mu_{k}}, \quad \mu_{k}^{-}=-i \sqrt{\mu_{k}},
$$

where $\mu_{k}$ (for $k \in \mathbb{N}_{0}$ ) is an eigenvalue of the operator $A_{0}$ with the corresponding eigenvector $\psi_{k}$. As for the eigenvalues $\mu_{k}^{+}$and $\mu_{k}^{-}$, they correspond to the eigenvectors $\psi_{k}^{+}$and $\psi_{k}^{-}$, respectively, where

$$
\psi_{k}^{+}=\left(\begin{array}{c}
\psi_{k} \\
i \sqrt{\mu_{k}} \psi_{k}
\end{array}\right), \quad \psi_{k}^{-}=\left(\begin{array}{c}
\psi_{k} \\
-i \sqrt{\mu_{k}} \psi_{k}
\end{array}\right) .
$$

We shall show that for no non-negative integer $k$

$$
\left\langle b_{0}, \psi_{k}^{+}\right\rangle_{\mathcal{H}}=0 \quad \text { or } \quad\left\langle b_{0}, \psi_{k}^{-}\right\rangle_{\mathcal{H}}=0 \text {. }
$$

Assuming that the above holds for some non-negative integer $k$, we obtain $\left\langle\bar{b}, \psi_{k}\right\rangle_{1}=0$ for that $k$. But then

$$
\psi_{k}=\frac{1}{\mu_{k}} A_{0} \psi_{k}=\frac{1}{\mu_{k}}\left(\widetilde{A} \psi_{k}+\left\langle\psi_{k}, \bar{b}\right\rangle_{1} \bar{b}\right)=\frac{1}{\mu_{k}} \widetilde{A} \psi_{k},
$$

so $\psi_{k}$ turns out to be an eigenvector $\widetilde{A}$ orthogonal to $\bar{b}$. This contradicts Part 1 of Theorem 2 .

Thus all the hypotheses of Theorem 5 are satisfied. After applying it, we only need to look closer at the formula for the control. We have

$$
\tilde{u}_{0}=-\left\langle Z, b_{0}\right\rangle_{\mathcal{H}}=-\langle\dot{Y}, \bar{b}\rangle_{1},
$$

hence $\tilde{u}=-\bar{b}^{+} Y+\tilde{u}_{0}=-\left\langle Y+\dot{Y},\left(I+\bar{b} \bar{b}^{*}\right)^{-1} \bar{b}\right\rangle_{\bar{H}}$. Moreover, for the system (29), we have $\|Y(t)\|_{4}$, $\|\dot{Y}(t)\|_{1} \rightarrow 0$ as $t \rightarrow \infty$ for any solution of (29). We apply Corollary 2 and immediately obtain

$$
\begin{array}{r}
\left\langle A\left(\begin{array}{c}
w(\cdot, t), \\
\xi(\cdot, t
\end{array}\right),\left(\begin{array}{c}
w(\cdot, t), \\
\xi(\cdot, t
\end{array}\right)\right\rangle+|\theta(t)|^{2} \rightarrow 0, \\
\left\|\left(\begin{array}{c}
\dot{w}(\cdot, t), \\
\dot{\xi}(\cdot, t
\end{array}\right)\right\|+|\dot{\theta}(t)|^{2} \rightarrow 0
\end{array}
$$


as $t \rightarrow \infty$. The above equations are equivalent to

$$
\begin{aligned}
\int_{0}^{1} K(x)\left|w^{\prime}(x, t)+\xi(x, t)\right|^{2} \mathrm{~d} x & \rightarrow 0, \\
\int_{0}^{1} E(x)\left|\xi^{\prime}(x, t)\right|^{2} \mathrm{~d} x & \rightarrow 0, \\
\int_{0}^{1}|\dot{w}(x, t)|^{2} \mathrm{~d} x & \rightarrow 0, \\
\int_{0}^{1} E(x)|\dot{\xi}(x, t)|^{2} \mathrm{~d} x & \rightarrow 0, \\
|\theta(t)|^{2} & \rightarrow 0, \\
|\dot{\theta}(t)|^{2} & \rightarrow 0 .
\end{aligned}
$$

The latter, together with the Friedrichs inequality imply (6). The proof of the theorem is complete.

\section{Conclusion}

The studying of control of a rotating beam with the aid of the torque is a natural consequence of a strictly theoretical problem of rotating beam control. The advantage of disregarding the torque is the possibility of finding the exact location of eigenvalues of the movement operator. Therefore solving the exact controllability problem is possible, because the appropriate Riesz basis is known. Moreover, dealing with the moment problem with known basic functions gives rise to the solution of the optimization problem. In the case studied here we do not even have an approximation of the eigenvalue locations. They may be placed anywhere in a (possibly wide) interval. Anyway, the existence of control with the aid of the torque is a consequence of the existence of control in a usual sense.

In contrast to the control problem, the stabilizability of the beam is the problem that is dealt with independently for the control with or without the aid of the torque. In this paper we have solved this problem for the case when the torque is applied.

\section{Acknowledgment}

The research of this paper was supported by the Polish Ministry of Science and Higher Education (grant no. N514 238 438).

\section{References}

Avdonin, S.A. and Ivanov, S.A. (1995). Families of Exponentials, Cambridge University Press, Cambridge.

Avdonin, S. and Moran, W. (2001). Ingham-type inequalities and Riesz bases of divided differences, International Journal of Applied Mathematics Computer Science 11(4): 803-820.
Kaczorek, T. (2012). Existence and determination of the set of Metzler matrices for given stable polynomials, International Journal of Applied Mathematics Computer Science 22(2): 389-399, DOI: 10.2478/v10006-012-0029-2.

Kato, T. (1966). Perturbation Theory for Linear Operators, Springer-Verlag, Berlin.

Krabs, W. and Sklyar, G.M. (2002). On Controllability of Linear Vibrations, Nova Science Publishers Inc., Huntington, NY.

Levin, B. (1961). On Riesz bases of exponential in $1^{2}$, Zapiski Matematicheskogo Otdieleniya Fiziko-matematicheskogo Fakul'teta Kharkovskogo Universiteta 27(4): 39-48.

Ostalczyk, P. (2012). Equivalent descriptions of a discrete-time fractional-order linear system and its stability domains, International Journal of Applied Mathematics Computer Science 22(3): 533-538, DOI: 10.2478/v10006-012-0040-7.

Paley, R.E.A.C. and Wiener, N. (1934). Fourier Transforms in the Complex Domain, American Mathematical Society, Providence, RI.

Respondek, J.S. (2008). Approximate controllability of infinite dimensional systems of the $n$-th order, International Journal of Applied Mathematics Computer Science 18(2): 199-212, DOI: 10.2478/v10006-008-0018-7.

Russell, D.L. (1967). Non-harmonic Fourier series in control theory of distributed parameter system, Journal of Mathematical Analysis and Applications (18): 542-560.

Sklyar, G.M. and Rezounenko, A.V. (2003). Strong asymptotic stability and constructing of stabilizing control, Matematitcheskaja Fizika, Analiz i Geometria 10(4): 569-582.

Sklyar, G.M. and Szkibiel, G. (2007). Spectral properties of non-homogeneous Timoshenko beam and its controllability, Mekhanika Tverdogo Tela (37): 175-183.

Sklyar, G.M. and Szkibiel, G. (2008a). Controllability from rest to arbitrary position of non-homogeneous Timoshenko beam, Matematitcheskij Analiz i Geometria 4(2): 305-318.

Sklyar, G.M. and Szkibiel, G. (2008b). Spectral properties of non-homogeneous Timoshenko beam and its rest to rest controllability, Journal of Mathematical Analysis and Applications (338): 1054-1069.

Sklyar, G.M. and Szkibiel, G. (2012). Approximation of extremal solution of non-Fourier moment problem and optimal control for non-homogeneous vibrating systems, Journal of Mathematical Analysis and Applications (387): 241-250.

Zerrik, E., Larhrissi, R. and Bourray, H. (2007). An output controllability problem for semilinear distributed hyperbolic systems, International Journal of Applied Mathematics Computer Science 17(4): 437-448, DOI: 10.2478/v10006-007-0035-y.

Grigory M. Sklyar is a full professor, affiliated to Szczecin University since 1997. He obtained his Ph.D. and qualification for an assistant professor from Kharkiv University. Currently, he is the head of the Control and Optimalisation Unit at the Institute of Mathematics. He is an author of over 70 research papers, mainly in system theory and control. He has taken part in several research projects: DAAD, a NATO Linkage grant, a KBN grant. He actively collaborates with professors from Ukraine (Kharkiv), Germany (Greifswald) and France (Nantes). 
Grzegorz Szkibiel received his Ph.D. from the State University of New York at Buffalo and has been affiliated to Szczecin University since 1988. $\mathrm{He}$ is with the Control and Optimalisation Unit in the Institute of Mathematics. He is an author of several research papers in system and control theories.

Received: 19 January 2013

Revised: 23 May 2013 